\title{
Formulation des consignes en FLE: étude des particularités linguistiques ${ }^{1}$
}

\section{(Elaboración deinstruccionesen Francés como lengua extranjera: estudio de particularidades lingüísticas)}

Jorge Pablo Barth Arroyo ${ }^{2}$

Universidad Nacional, Costa Rica

\section{résumé}

Les enseignants de FLE affrontent des difficultés au moment de combiner les courants européens en évaluation et en docimologie, avec les directives du MEP par rapport aux épreuves pour les compétences écrites, spécifiquement dans la rédaction des consignes. Cette étude triangule un diagnostic du projet PROFE de l'UNA en 2005, une enquête et un instrument appliqué en 2010 à un groupe de 40 enseignants. A la suite de cette étude, on offre un module d'autoformation pour les enseignants et on réalise un pilotage pour sa mise en pratique lors d'une formation de PROFE.

\section{resumen}

El profesorado de Francés como lengua extranjera enfrenta dificultades al combinar los postulados de las tendencias europeas en evaluación y docimología con las directrices oficiales, en cuanto a las pruebas para las

$T$ Elaborado con base en la ponencia presentada por el autor en el IV Congreso Internacional de Lingüística Aplicada, llevado a cabo en mayo de 2013, en el Campus Omar Dengo, de la Universidad Nacional de Costa Rica. Recibido: 19 de mayo de 2012; aceptado: 30 de agosto de 2012. Titre original «Les particularités linguistiques dans la formulation des consignes: analyses syntaxique et sémantique».

2 Escuela de Literatura y Ciencias del Lenguaje. Correo electrónico: jopaba2@ @otmail.es

$$
L_{\text {etras }} 52 \text { (2012), ISSN 1409-424X }
$$


competencias escritas, específicamente en la redacción de instrucciones. Este estudio triangula un diagnóstico realizado por el proyecto PROFE de la UNA en 2005, una encuesta y un instrumento aplicados en 2010 a un grupo de 40 docentes. Se propuso un módulo correctivo para docentes y se ejecutó un plan piloto para su implementación como parte de la formación continua de PROFE.

Mots-clés: évaluation en FLE, erreurs, analyses linguistique

Palabras clave: evaluación en FLE, errores, análisis lingüístico

\section{Introduction}

L'apprentissage du français en tant que langue étrangère (FLE) ne remonte pas aux dernières années de l'histoire de l'éducation costaricienne. Il existe depuis plus de 150 ans avec les premiers cours au Lycée de Santo Tomás en 1824 et l'arrivée des Sœurs de Sion en 1869 , mais il ne devient une matière officielle du curriculum en secondaire qu'en 1909.

Aujourd'hui, le Costa Rica expérimente un changement rapide au niveau culturel et social et les gens s'intéressent de plus en plus à l'apprentissage des langues étrangères car l'ancienne idée d'un pays monolingue où l'on ne s'exprime qu'en espagnol a été laissée dans le passé. Dans les dernières années, l'horizon s'étend et le besoin d'un réel plurilinguisme apparaît chaque fois plus clair. Des domaines nouveaux pour l'emploi des langues étrangères s'ouvrent et plus que jamais, donner aux élèves costariciens l'accès à un vrai outil de travail se confirme comme un besoin du système éducatif. Actuellement, le français est enseigné dans quelques écoles primaires, universités, académies de langues telles que l'Alliance Française ou le Centre de Langues Berlitz mais principalement au secondaire. Il y a environ huitcent enseignants de FLE et trois-cent mille élèves qui étudient cette langue sous le système du Ministère de l'Education Publique (MEP).

Nonobstant, les professeurs de français de notre pays sont souvent confrontés à une contradiction entre les courants européens en 
évaluation et en docimologie -Tagliante (2005), Courtillon (2003), le Cadre Européen Commun de Référence pour les Langues, CECRL (2001) -et les directives du MEP concernant les épreuves écrites, spécifiquement dans la formulation des consignes.

D'une part, les référentiels proposés aux enseignants de langues tels que le CECRL, décrivent de manière détaillée des connaissances et des compétences acquises en langue dite «étrangère». Il s'agit d'un document important qui parle d'évaluation en termes certificatifs, c'est-à-dire, en termes de résultats finaux après un processus, mais il ne propose pas de suggestions concrètes pour la partie formelle des épreuves. Il est donc, nécessaire de faire une approche de notre réalité vécue dans les salles de classe de notre pays car le contexte du FLE au niveau européen (et des autres langues étrangères enseignées dans chaque système scolaire) est diamétralement opposé à notre contexte.

D'autre part, le MEP n'offre pas de documents concernant l'évaluation des apprentissages pour les langues étrangères. Sauf, un ancien document sur l'évaluation spécifique de l'anglais (mis en cause par le Département d'Evaluation), on ne peut pas trouver des directives spécifiques aux cours et les versions des règlements d'évaluation des dernières années changent peu et ils s'occupent principalement des aspects formels de l'évaluation sommative.

\section{Axes de l'étude}

Cette recherche est basée sur trois axes: une étude diagnostique sur la manière dans laquelle les enseignants de FLE rédigent habituellement les consignes dans leurs épreuves écrites, un dispositif virtuel d'autoformation dirigé aux professeurs de FLE en cohérence avec les directives actuelles du MEP et finalement l'évaluation de la mise en pratique du dispositif lors d'une formation du projet PROFE à l'UNA et ses résultats. 


\section{L'importance des consignes}

Tout enseignant souhaite que les apprenants réalisent correctement le travail, devoir, activité de classe ou évaluation qu'il avait conçu mais, que se passe-t-il quand les résultats ne sont pas ceux qu'il attendait au début? Il n'est pas facile de répondre à cette question, car il y a plusieurs phénomènes qui interviennent dans le processus de la réception et l'expression dans une langue étrangère, spécifiquement dans la rédaction d'une consigne. Avant de continuer, il est important de définir le terme consigne. Celui-ci constitue un synonyme du mot instruction. Pour Reaynal et Rieunier ${ }^{3}$, il désigne «un ordre donné pour faire effectuer un travail. Enoncé indiquant la tâche à accomplir ou le but à atteindre».

La consigne peut être orale ou écrite. Dans le premier cas, l'apprenant peut percevoir des signes externes tels que les gestes, l'intonation et le volume de la voix qui lui permettent de mieux comprendre la tâche à réaliser. De plus, l'enseignant peut reformuler la consigne si nécessaire, en utilisant un vocabulaire moins formel.

Par contre, la consigne écrite demande plus d'attention à l'écriture, car un mot mal écrit peut confondre l'apprenant. Les signes de ponctuation peuvent varier le sens d'une phrase et permettre à l'apprenant de distinguer entre une affirmation et une question.

En outre, quand on s'exprime par écrit, les mots ne viennent pas si vite à l'esprit que quand on parle, on n'utilise pas le même langage et on ne coupe pas non plus certains mots ou structures grammaticales telles que le «ne» dans la négation. En plus, d'autres signes externes peuvent contribuer ou nuire à la compréhension de la consigne, la taille et le type de lettre, la qualité du papier et de l'impression sur le papier ou l'utilisation d'une image par exemple. Soit à l'oral ou à l'écrit, l'important est que le sujet comprenne correctement les actions qu'il doit exécuter.

3 F. Reynal et A. Rieunier, Pédagogie: dictionnaire des concepts clés (Paris: ESF éditeur, 1997) 90. 
La consigne constitue une partie essentielle de l'épreuve, car «de la qualité de la consigne dépend en partie la qualité du travail effectué» Raynal et Rieunier (1997).

On pourrait même dire qu'un item manque de clarté, de précision et de sens s'il n'est pas accompagné de la bonne consigne. L'enseignant ne doit pas oublier que la consigne doit faciliter à l'apprenant la compréhension de la tâche au moment de l'évaluation. Veltcheff et Hilton ${ }^{4}$ exposent que «la consigne n'est pas un obstacle mais bien un guide qui permette réaliser correctement ce qui est demandé».

Nonobstant sa rédaction est une activité qui mérite toute l'attention de la part de l'enseignant. Raynal et Rieunier ${ }^{5}$ expliquent que le fait de rédiger des consignes dans une épreuve écrite n'est pas une tâche facile car le travail, la conception de l'idée chez les apprenants et l'exécution de l'action demandée ou l'objectif atteint englobe un grand nombre des variables ou des facteurs qui peuvent intervenir dans le processus, c'est pourquoi, il est objet d'étude de quelques sciences telles que la docimologie, la psychologie cognitive et la linguistique du discours. Donc, l'enseignant doit prendre en compte l'écriture, les éléments implicites et socioculturels, ainsi que la perception du monde que possède l'apprenant.

Le Cadre européen commun de référence pour les langues affirme que «des consignes simples, pertinentes et suffisantes (ni trop ni trop peu d'information et un guidage non verbal approprié) réduisent la possibilité de confusion en ce qui concerne la démarche et les objectifs ${ }^{6}$. Donc, la consigne peut être courte ou longue si l'on a besoin de plus d'explications, comme par exemple, dans le cas d'un essai ou d'une composition. La consigne ne peut être ni si courte ou vague que l'apprenant ne sache quelle action accomplir, ni si longue qu'elle puisse distraire l'attention de l'élève. La règle d'or: elle sera

\footnotetext{
4 C. Veltcheff et S. Hilton, Evaluation en FLE (Paris: Hachette, 2003) 58.

5 Reynal et Rieunier, 95.

6 Conseil de L'Europe, Division des politiques linguistiques, Strasbourg, Cadre européen commun de référence pour les langues: apprendre, enseigner, évaluer (Paris: Didier, 2000) 126.
} 
simple et claire, avec la transparence possible (favorisant un transfert positif de la langue maternelle vers la langue cible).

Ce transfert est expliqué par Courtillon ${ }^{7}$ à travers les opérations cognitives qui favorisent la compréhension et l'acquisition d'une nouvelle langue telles que la perception et la mémorisation. Dans la première, l'élève commence à s'approprier des codes de communication tels que le lexique et la morphosyntaxe. Dans cette étape il pourra découvrir par exemple la signification des consignes des exercices et des activités de classe. Il les comparera avec sa langue maternelle afin de trouver des éléments connus ou des ressemblances afin de codifier le message dans son esprit. Pendant l'étape de la mémorisation, l'élève tentera de mémoriser la consigne et de l'associer au type d'item ou activité présentée par l'enseignant. S'il ne peut pas se souvenir de toute l'instruction, il se rappellera au moins du verbe utilisé et de sa signification. C'est pourquoi l'importance de la perception et la mémorisation des bonnes consignes en FLE.

D'autre part, R. Torres ${ }^{8}$, chef du département d'évaluation des apprentissages au MEP, propose aux enseignants de «rédiger des consignes générales et spécifiques de manière à ce qu'elles orientent l'apprenant par rapport à la tâche à suivre au moment de résoudre l'épreuve».

\section{L'étude du diagnostic}

Cette étude a été réalisée grâce à la collaboration du Projet Français et Education (PROFE), car il a été inspiré dans les résultats d'un diagnostic national que ce projet avait élaboré pendant les années 2004 et $2005^{9}$ où les enseignants de FLE de notre pays montraient leur intérêt à mettre à jour leurs connaissances en évaluation des apprentissages, spécialement par rapport aux consignes

7 J. Courtillon, Elaborer un cours de FLE (Paris: Hachette, 2003) 27-30.

8 R. Torres, La prueba escrita (traduction littérale) (San José : MEP, 2010) 5.

9 M. Núñez et M. Vargas, Diagnóstico preliminar sobre la formación profesional de los profesores de Francés en Costa Rica (PROFE, UNA, 2004); et Diagnóstico nacional sobre la situación de la enseñanza del francés en Costa Rica (PROFE, UNA, 2005). 
des épreuves. Ensuite, on a enquêté la coordinatrice du projet pour vérifier les conclusions des diagnostics précédemment élaborés et confirmer l'orientation de la recherche et sa configuration, à partir de questions à réponse ouverte ou restreinte et une grille pour le choix des thèmes d'intérêt.

Lors d'un séminaire de l'Association Nationale de Professeurs de Français, ACOPROF en décembre 2010, quelques professeurs de français ont volontairement participé dans une enquête sous forme de test diagnostic avec des activités pratiques, afin de mieux comprendre la perception, les habitudes et la pratique quotidienne des consignes à travers l'analyse des catégories grammaticales et des procédés sémantiques et syntaxiques, restreintes à la formulation de consignes pour la réception et à la production écrites.

Ce diagnostic a montré que de 40 enseignants enquêtés, la totalité du groupe a manifesté l'inquiétude pour l'élaboration des épreuves écrites et orales. Face à la question «Quels sujets aimeriez-vous aborder pendant le module d'autoformation?» posée dans l'instrument, il faut dire que 36 professeurs ont choisi la création d'items et 33 se sont intéressés à la rédaction de consignes pour les épreuves. Ce résultat a confirmé le souhait constant de la part des enseignants à être formés dans le domaine de l'évaluation et la création des épreuves orales et écrites.

\section{Particularités linguistiques sur la rédaction des consignes}

Un instrument sous forme de test a été proposé aux enseignants lors d'une formation de FLE en 2010 afin de recenser les pratiques habituelles et la formulation des consignes en français que les enseignants utilisent normalement dans leurs épreuves ou activités écrites. Il faut souligner que tous ces participants ont volontairement collaboré avec cette étude diagnostique.

A partir de la catégorisation des réponses, on a analysé les aspects sémantiques et syntaxiques des résultats. Les données de cette 
recherche ont servi de base pour démontrer la manière dans laquelle les enseignants traduisent au français les consignes les plus utilisées en espagnol en cohérence avec les différents types d'items proposés par le MEP dans les épreuves écrites. Les phrases proposées sont très souvent utilisées comme des consignes en espagnol, mais leur traduction au français n'est jamais littérale, ce qui pose des difficultés aux enseignants moins formés ou qui maîtrisent moins les aspects culturels relatifs aux consignes en français.

Pour bien évaluer les apprenants il est nécessaire d'assurer que la construction des épreuves garantisse la bonne formulation linguistique des consignes, c'est pourquoi on a réalisé une analyse sémantique et syntaxique des principales consignes utilisées dans le test diagnostic. On verra ensuite les résultats reflétés à partir de cetteétude.

\section{Etude sémantique sur la formulation des consignes}

La sémantique peut se définir comme «la discipline qui cherche à expliciter les propriétés des formes linguistiques leur permettant ultimement d'évoquer l'univers d'expérience $\rangle^{10}$. On est parti de ce principe pour analyser les réponses des enseignants collaborateurs dans le diagnostic. Voici quelques exemples:

Premier exemple: «Marque con equis la respuesta correcta.» Cette consigne correspond à un item à choix multiple (QCM) où l'apprenant doit choisir l'option qui s'adapte le mieux à l'énoncé ou à la question posée. On peut proposer deux équivalents au niveau de FLE: «Cochez la bonne réponse» ou «Cochez la réponse correcte». Par contre, on a remarqué que la plupart des enseignants qui ont utilisé le verbe «cochez» ont ajouté à leur consigne que c'était «avec un $\mathrm{X}$ » ou «d'un $\mathrm{X}$ » ce qui montre une répétition, car le verbe «cocher» suppose déjà le fait de marquer d'un trait la bonne réponse. De plus, au niveau culturel, on peut dire qu'au Costa Rica, les apprenants

10 P.Larrivée, Une histoire de sens. Panorama de la sémantique linguistique depuis Bréal (Bruxelles: P.I.E. Peter Lang, 2008) 9. 
sont habitués à cocher spécifiquement en utilisant un «X» la bonne réponse dans ce genre d'item.

En ce qui concerne le verbe employé dans cette consigne, on a constaté que certains enseignants ont utilisé des verbes à l'impératif dont le sens ne fait aucune référence à l'évaluation des apprentissages, par exemple, les verbes «couchez» et «clochez».

Le premier possède plusieurs significations. Premièrement, mettre au lit quelqu'un, expression utilisée pour les enfants comme «Juliette couche ses enfants à $20 \mathrm{~h}$ ». Il désigne aussi un nom «le coucher du soleil». Par contre, accompagné de la préposition «avec» il change complètement son sens car, cela veut dire en langage argotique, avoir des rapports sexuels avec quelqu'un. Si bien, le verbe existe en français, il n'y a aucune cohérence avec l'évaluation de FLE. Imaginez les réactions de quelques adolescents s'ils se rendent compte du caractère sexuel que possède ce verbe. Donc, utiliser ce verbe peut provoquer des quiproquos avec les apprenants et même des problèmes légaux avec les parents d'élèves. Par rapport à la signification du verbe «clocher», on en distingue trois: un objet, «le clocher d'une église», boiter ou trainer une jambe et aller de travers, c'est-à-dire, quelque chose qui ne fonctionne pas correctement. En somme, les trois significations deviennent inutiles pour la consigne souhaitée.

Par rapport à la consigne «Cochez la solution correcte», proposée par certains enseignants est peut être bien formulée linguistiquement s'il s'agit d'un item où l'on demande à l'élève de résoudre un problème mathématique ou scientifique.

Le deuxième exemple de consigne est «Complete los siguientes enunciados en los espacios en blanco». Elle accompagne un item à réponse courte $(\mathrm{QRC})$ où l'option attendue de la part de l'apprenant termine une phrase inachevée. La plupart des enseignants ont choisi le verbe «compléter» pour cette consigne mais, il faut souligner qu'en français on pourrait aussi utiliser le verbe «remplir» si c'était le cas d'un formulaire, questionnaire ou fiche d'identité. 
En outre, on a trouvé de consignes qui manquent de clarté au niveau d'évaluation des apprentissages, soit qu'elles sont trop longues qu'elles puissent distraire l'apprenant et devenir ambiguës, soit qu'elles se présentent à l'élève de manière trop vague qu'elles manquent d'information précise pour exécuter l'action demandée et laissent à l'élève le travail de deviner ce que le professeur attend de lui. On peut citer l'exemple de la consigne en espagnol «Escriba en el paréntesis de la columna A el número de la columna B que le corresponde» où l'on a lu des réponses telles que «Il y a la colonne a et la colonne b. Associez-les !» Pour ce type d'item, il existe deux sous-catégories: l'item d'association simple et multiple. La différence réside dans le nombre de correspondances entre chaque élément des deux colonnes. Il est toujours indispensable de présenter à l'apprenant le sujet à développer dans l'exercice et décrire dans les consignes le contenu de chacune des colonnes et indiquer si chaque réponse pourra être utilisée une ou plusieurs fois dans la colonne contraire (Torres) ${ }^{11}$. De plus, il faut dire que le MEP n'accepte plus l'item d'association multiple dans les épreuves écrites, même si on le trouve dans quelques manuels ou méthodes de langues, les enseignants peuvent s'en servir seulement pour certaines activités de classe ou devoirs. Ainsi donc, la consigne proposée antérieurement est imprécise et on utilise un type de langage informel. Il ne faut pas oublier quand on rédige des consignes qu'une épreuve est un document officiel.

Parmi les particularités dans la formulation des consignes trouvées, on a remarqué des problèmes au niveau sémantique concernant l'emploi des prépositions. Il est vrai que seule l'exposition à la langue française permet d'atteindre la maitrise dans l'utilisation de certaines prépositions, car la langue évolue et adopte des structures linguistiques de caractère culturel et non grammatical. Entre les formulations erronées les plus communes des enseignants, on peut mentionner:

$\Pi$ R. Torres, La prueba escrita (San José: MEP, 2010). 
Tableau 1. Ce tableau montre quelques exemples de l'emploi des prépositions de la part des enseignants et leur utilisation correcte.

\begin{tabular}{|c|c|c|}
\hline Préposition & Faute & Emploi \\
\hline sur $\neq$ dans & Ecrivez sur l'espace... & Ecrivez dans l'espace \\
\hline dans $\neq$ pendant & $\begin{array}{c}\text {..ce que vous ferez } \\
\text { dans les vacances }\end{array}$ & $\begin{array}{c}\text {...ce que vous ferez } \\
\text { pendant les vacances }\end{array}$ \\
\hline à $\neq$ par & A l'écrit & Par écrit \\
\hline en $\neq$ dans & Mettez en la parenthèse & $\begin{array}{c}\text { Mettez dans la paren- } \\
\text { thèse }\end{array}$ \\
\hline $\begin{array}{c}\text { Absence de } \\
\text { «ans le }(\text { gérondif) }\end{array}$ & $\begin{array}{c}\text { Rédiger un paragraphe } \\
\varnothing \text { indiquant }\end{array}$ & $\begin{array}{c}\text { Rédiger un paragraphe } \\
\text { en indiquant }\end{array}$ \\
\hline comme $\neq$ au & Comme minimum & Au minimum \\
\hline
\end{tabular}

On peut dire aussi qu'il y a une interférence de l'espagnol dans la rédaction des consignes, ce qui est commun chez les apprenants d'une langue étrangère mais, qui doit, en effet, être minimisée aufur-et-à-mesure que l'on étudie le français.

D'ailleurs, quelques enseignants ont eu des problèmes dans la formulation de la consigne «Observe el siguiente personaje y descríbalo físicamente. Utilice al menos cinco oraciones completas y elementos variados» dont l'équivalent en français est «Regardez le personnage et décrivez-le physiquement. Utilisez au moins cinq phrases complètes avec des éléments variés».

L'une des formulations erronées analysées dans cette consigne était «observez-vous...» et «regardez-vous» que même s'il est bien écrit, change complètement le sens de la consigne car, elle demande à l'apprenant de réaliser une description de lui-même et non du personnage présenté dans l'épreuve, ce qui provoque un doute sur l'action à réaliser. Enfin, l'élève pourra utiliser la logique pour discerner que le personnage possède un rôle plus important dans l'épreuve et 
il le décrira. Néanmoins, il y aura peut-être des élèves qui feront leur propre portrait physique et qui suivront la consigne écrite.

Des structures similaires ont été trouvées dans la consigne «Transforme las siguientes frases al imperfecto del modo indicativo». On propose cette traduction en français «Mettez les phrases suivantes à l'imparfait de l'indicatif». Pourtant, on a trouvé des réponses telles que: «Transformez les phrases suivantes de l'imparfait à la manière indicatif». Cette consigne manque de sens logique, car le temps de l'imparfait appartient déjà au mode de l'indicatif. Donc, l'élève sera confondu de ne pas savoir ce qui représente pour l'enseignant «la manière indicatif» En plus, cette consigne indique la présence des phrases rédigées à l'imparfait, ce qui ne serait pas réel dans l'épreuve, car la consigne demandait à l'élève -en principe-de transformer ou de mettre les phrases à l'imparfait.

Une autre traduction de la même consigne en français est «Transformer les phrases à l'impératif». Tout d'abord, il faut souligner que l'impératif est un temps verbal tout à fait différent à ceux qui appartiennent à l'indicatif. Autrement dit, il s'agit d'un temps à part, comptant deux formes verbales, l'impératif présent comme dans la phrase «Voyons le nouveau film» et l'impératif passé «Ayons vu le nouveau film». Ainsi donc, le fait de mettre les phrases à l'impératif change complètement le sens de la consigne, raison pour laquelle l'élève réalisera une action tout à fait différente à celle qui avait été conçue au début par l'enseignant.

En somme, il est évident que les traductions littérales des consignes faites par les enseignants peuvent nuire à la réception des écrits en FLE chez les apprenants, situation qui rend difficile le processus d'appropriation des connaissances des élèves et leur évaluation. Vécchi ${ }^{12}$ réfléchit à ce sujet et il nous indique que souvent la confusion sur les tâches à réaliser n'est pas toujours la faute des apprenants sinon de nous-mêmes car «lorsqu'un enseignant demande

12 G. Vécchi, Aider les élèves à apprendre (Paris: Hachette, 2000). 
à des apprenants de produire un travail et qu'il n'obtient pas les résultats espérés, ce ne sont pas toujours les élèves qui sont en cause. Parfois, lui aussi a sa part de responsabilités!».

\section{Etude syntaxique sur la formulation des consignes}

En ce qui concerne l'étude syntaxique, on a analysé les mêmes réponses par rapport aux consignes et la même population de professeurs. Par rapport à la syntaxe, on peut la définir comme l'étude de la forme et la relation qui existe entre les mots pour créer une phrase ou un énoncé. L'analyse syntaxique est basée sur trois aspects ou catégories, le mot (lexique et groupes lexicaux), le syntagme ou groupe de mots à l'intérieur d'une phrase et bien sûr, la phrase qui comprend et qui met en ordre tous les éléments intérieurs en sens logique. Après la lecture de chacune des réponses des collaborateurs du diagnostic, nous pouvons affirmer que les professeurs ont formé, en général, des phrases correctes quant à leur structure linguistique, c'est-à-dire, que les phrases créés possèdent une certaine cohérence morphosyntaxique. Elles comportent tous les éléments grammaticaux nécessaires pour avoir un sens logique, même si l'on peut trouver beaucoup de fautes. Pourtant, cela ne veut pas dire, que dans le domaine de l'évaluation, les consignes soient complètement claires et précises. Entre les formulations des sujets de l'étude, on peut citer l'absence d'un élément ou mot dans la phrase «Ecrivez un (x) la réponse correcte». On peut supposer que l'élément qui manque pourrait être une préposition. Par contre, le fait de deviner l'action à réaliser ne doit pas être prise en charge par l'apprenant.

Dans certaines réponses on a constaté une pronominalisation de verbes: Etant donné: «Cochez-vous avec une «x» la réponse correcte».

Il est difficile de deviner ce que voudrait dire cette consigne ou s'il s'agit plutôt d'une question sans point d'interrogation, mais il est évident que cette phrase peut confondre l'élève au moment de résoudre l'item. 
Par rapport à l'exemple de consignes «Complete los siguientes enunciados en los espacios en blanco», on a constaté une grande influence de la syntaxe espagnole sur la syntaxe française. Autrement dit, les professeurs calquent des structures purement syntaxiques de l'espagnol et ils les traduisent mot à mot en langue française ${ }^{13}$. Pour illustrer cet argument, on peut citer quelques structures telles que l'inversion des noms et d'adjectifs qualificatifs: «Complétez les suivants énoncés dans les espaces en blanc». D'autres expressions calquées de l'espagnol et une redondance dans les consignes se présente dans les phrases: «Complétez les énoncés dans les espaces donnés» ou «Complétez les énoncés dans les espaces que sont en blanc».

Les changements d'ordre des mots dans une phrase peuvent varier la consigne. Dans l'exemple «Complétez les blancs avec les énoncés suivants» suppose qu'il faut choisir l'énoncé qui complète chacune des phrases proposées, comme s'il y avait une banque de mots. Cependant, l'objectif au début était que l'élève aurait construit la fin de l'énoncé qui complète la phrase.

En outre, si l'on observe la manière d'écrire les mots, on a trouvé beaucoup de fautes d'orthographe telles que le manque d'accords en genre et nombre des noms ou d'adjectifs qualificatifs. Voici quelques exemples: «espaces en blanche», «espac en blanch», «les espace», «phrases suivants», «spas en blanc», «le personnaje proposé», «lignes en blanche», «les énoncie suivants» et «fisiquement». D'autres fautes d'orthographe trouvées concernent l'absence constante d'accents aigus, graves et circonflexes, surtout dans les verbes. Par exemple : «repondez, completez ou decrire».

L'utilisation de termes ne concernant pas l'évaluation sont aussi communs dans les consignes, parmi lesquels on peut mentionner «space» comme le space sidéral et «spas» au lieu d'«espace», ce qui met en évidence le manque de vocabulaire chez certains enseignants. On a même constaté des problèmes avec l'emploi des déterminants

13 Cette recherche a absolument conservé la fidélité des réponses apportées par les participants. 
possessifs et des pronoms possessifs. La phrase «...votre futures vacances» où l'on utilise le déterminant possessif «votre» au lieu du pronom possessif «vos» en constitue un exemple.

Dans très peu de cas, on a lu des phrases dont la conjugaison des verbes employés ne correspond pas aux personnes utilisées. Tel est le cas des phrases : «...ce que je fera pendant les vacances» ou «ce que tu ferez pendant les vacances».

\section{Elaboration du dispositif virtuel d'autoformation}

Suite à l'analyse linguistique sur la rédaction des consignes de la part des enseignants et leurs principales inquiétudes en matière d'évaluation on a élaboré un module virtuel d'autoformation pour les professeurs de FLE. Ce dispositif était censé être capable de suppléer à quelques besoins exprimés par les professeurs de FLE du Costa Rica. On a envisagé la création d'un matériel innovateur susceptible de mettre en contexte quelques notions en évaluation, spécialement les consignes, avec les directives du Ministère de l'Education $\mathrm{Pu}$ blique de notre pays.

Le dispositif est composé de huit fiches, accompagnées de la théorie de chaque sous-thème, des références bibliographiques au pied de page pour faciliter la consultation immédiate lors du module.

Dans la première partie du module, on a fait une approche théorique sur l'évaluation de la réception et l'expression écrite au Costa Rica permettant à l'apprenant d'avoir une notion plus claire et précise des principaux concepts de base et quelques théoriesconcernant la docimologie et l'évaluation des apprentissages.

Pour la deuxième partie, on a présenté une description d'une épreuve écrite ainsi que les principales directives établies par le MEP au moment de rédiger une épreuve. On a étudié quelques règlements et des lois qui servent de guide aux enseignants qui construisent des épreuves écrites au Costa Rica. Dans cette partie, on a décrit quelques documents officiels et lois en vigueur par rapport à l'évaluation des 
apprentissages tels que le Règlement de l'évaluation des apprentissages du MEP et «l'épreuve écrite».

D'ailleurs, il faut souligner que chaque sujet étudié dans le module a été accompagné d'une fiche pédagogique afin de compléter l'apprentissage du professeur. Chaque fiche est composée du nom du thème en étude, les objectifs spécifiques à atteindre ainsi que des exercices pratiques à résoudre afin de tester ses connaissances. A la fin du dispositif, les enseignants seront capables de confectionner une épreuve écrite à partir d'une table de spécifications et en proposant les items et les consignes qui les accompagnent.

\section{Résultats de la mise en pratique du dispositif virtuel}

Une partie du dispositif virtuel a été mis en pratique en décembre 2011 lors d'un séminaire national de professeurs de français à l'UNA. Les participants étaient des enseignants actifs dans le système éducatif costaricien au niveau public et privé qui se sont volontairement inscrits à cette formation. Ce séminaire était composé de deux parties, l'une sur place au campus Omar Dengo, à l'UNA et l'autre de caractère virtuel, laquelle était composée en même temps d'une partie A et B, il faut indiquer que la partie $\mathrm{B}$ est celle qui correspond à notre dispositif en question.

On a donc choisi pour ce séminaire la première partie du dispositif concernant la docimologie et ses buts, l'évaluation et ses fonctions ainsi que les types les plus utilisés au Costa Rica. Il avait une valeur totale de 38 points et 26 enseignants s'y sont inscrits volontairement. Ils ont résolu les fiches pédagogiques et ils ont été évalués par des fonctionnaires de l'UNA qui travaillent au projet PROFE.

Après la formation, on a analysé les résultats des participants et on a pu constater que la meilleure note obtenue par les enseignants c'est un 37/38. C'est-à-dire, un 97\% du dispositif. De plus, la deuxième meilleure note réussie par deux personnes est de 36/38.

Par contre, la moindre note obtenue, c'est 29/38 et il y a deux participants qui l'ont eue, ce qui constitue la réussite d'un $76 \%$ du dispositif $\mathrm{B}$ du séminaire virtuel. 
En outre, on peut remarquer que «la mode» ou la note qui se répète le plus chez les participants est de 33/38. Il faut dire que la moyenne du groupe d'enseignants est également de 33/38.

Ainsi donc, le comportement normal des gens par rapport aux notes représente la réussite d'un $87 \%$ du dispositif. C'est un pourcentage considérable qui met en évidence un succès chez la totalité des professeurs participants.

\section{Conclusions}

En résumé, la manière dans laquelle les enseignants rédigent les consignes dans une épreuve, activité de classe ou devoir peut interférer avec le processus de réception, mémorisation et production des apprenants. Les consignes dans les épreuves de FLE montrent un point faible chez les enseignants. Il faut dire que cette étude sur les consignes est le premier de ce type, réalisé dans notre pays.

Les problèmes de rédaction, syntaxiques et sémantiques, dans les consignes et les items qui les accompagnent, reflètent les déficiences linguistiques des enseignants dans la construction des phrases, des énoncés et des évaluations.

Il est nécessaire que les apprenants s'habituent aux consignes qu'ils peuvent trouver dans les manuels et les épreuves en FLE.

Afin d'éviter l'ambigüité dans les épreuves écrites en FLE, il faut mettre à la portée des enseignants plus d'informations linguistiques correctes pour la rédaction des consignes en français en harmonie avec les directives du MEP. La mise en place du projet peut vraiment changer la vision que possèdent les enseignants par rapport à la réception et la compréhension d'écrits chez les apprenants afin d'éviter l'ambigüité dans les épreuves de français. 